\title{
Resultados de la desviación vertical disociada posterior a la cirugía en el Instituto de Oftalmología Fundación de Asistencia Privada Conde de Valenciana IAP
}

\section{Results in dissociated vertical deviation after surgery in the Instituto de Oftalmología Fundación de Asistencia Privada Conde de Valenciana IAP}

\author{
Diana E. Jiménez-Guerrero ${ }^{*}$, A. Jéssica Vargas-Ortega ${ }^{1}$, Lucrecia Berganza-Canales ${ }^{1}$, \\ Aída Jiménez-Corona ${ }^{2}$ y Claudia E. Murillo-Correa ${ }^{1}$ \\ ${ }^{1}$ Departamento de Estrabismo; ${ }^{2}$ Departamento de Epidemiología y Estadística. Instituto de Oftalmología, FAP Hospital Conde de Valenciana, IAP, \\ Ciudad de México, México
}

\section{Resumen}

Objetivo: Determinar el resultado posterior a la cirugía de la desviación vertical disociada (DVD) descompensada en el Instituto de Oftalmología Fundación de Asistencia Privada Conde de Valenciana entre los años 2004 y 2014. Materiales y métodos: Estudio ambispectivo en el que se evaluó el resultado a largo plazo de la cirugía para la corrección de la DVD en 40 pacientes de la institución. Variables de interés: Capacidad visual y presencia de ambliopía, patología estrabológica asociada, magnitud preoperatoria de la desviación, cirugía realizada, resultado de la cirugía, requerimiento de más cirugías y tiempo de seguimiento. Resultados: Se estudiaron 40 pacientes, de los cuales 28 eran del sexo femenino y 12 eran del sexo masculino; el promedio de edad en el momento de la cirugía fue de 9.6 años, con moda de 4 años (rango 3-35 años). La patología estrabológica asociada más frecuente fue la endotropía no acomodativa (60\%), seguida de la desviación horizontal disociada (32.5\%). Se realizó retroinserción de ambos rectos superiores en el $77.5 \%$, plegamiento de los rectos inferiores en el 15\%, y transposición anterior de oblicuos inferiores en el 7.5\%. Doce pacientes presentaron ambliopía, correspondiente al 30\%. Posterior a la cirugía, el $82 \%$ presentó compensación de la DVD, solo tres pacientes requirieron cirugías adicionales. El tiempo de seguimiento promedio fue de 4.8 años (rango de 1 a 10 años). Conclusión: La mayoría de los pacientes operados de DVD descompensada en la institución presentan compensación de la misma, posterior a la cirugía. La cirugía más realizada en la institución para tratamiento de la DVD es la retroinserción de rectos superiores.

Palabras clave: DVD descompensada. Resultados retroinserción recto superior. Compensación.

\section{Abstract}

Objectives: To determine the results after decompensated DVD surgery at the Instituto de Oftalmología, Fundación de Asistencia Privada "Conde de Valenciana," during the 2004-2014 period. Materials and Methods: Ambispective study, in which the long-term results of DVD-correcting surgery on 40 patients of our Institute were evaluated. Variables of interest: Best-co-

Correspondencia:

*Diana E. Jiménez-Guerrero

La Quemada 45, int 4

Col. Narvarte Oriente, Del. Benito Juárez Fecha de recepción: 31-05-2018

Disponible en internet: 01-03-2019 C.P. 03020 , Ciudad de México, México

Fecha de aceptación: 28-11-2018 Rev Mex Oftalmol. 2019;93(2):57-62 E-mail: dejg82@yahoo.com DOI: 10.24875/RMO.M18000060 www.rmo.com.mx 0187-4519/@ 2018 Sociedad Mexicana de Oftalmología. Publicado por Permanyer México. Este es un artículo Open Access bajo la licencia CC BY-NC-ND (http://creativecommons.org/licenses/by-nc-nd/4.0/). 
rrected visual acuity, presence of amblyopia, associated strabismus, pre-surgical magnitude of the deviation, type of surgery, surgical result, need for additional surgeries, and follow-up time. Results: Forty patients were studied, of which 28 were female and 12 were male; average age at surgery was 9.6 years, with a mode of 4 years (range 3-35 years). The most frequent associated strabismus was congenital esotropia (60\%), followed by dissociated horizontal deviation in $32.5 \%$. Superior rectus recession was performed in $77.5 \%$ and inferior rectus tucking in $7.5 \%$. Twelve patients had amblyopia (30\%). After surgery, $82 \%$ showed DVD compensation and only 3 patients required additional surgeries. The average follow-up period was 4.8 years (range 1-10). Conclusion: Most patients that underwent surgery for decompensated DVD showed compensation after surgery. Superior rectus recession is the most commonly performed surgery for the treatment of DVD.

Key words: Decompensated DVD. Superior rectus recession results. Compensation.

\section{Introducción}

La desviación vertical disociada (DVD) es un fenómeno bilateral que presenta un movimiento de elevación, abducción y exciclotorsión cuando el examinador ocluye uno de los ojos, y luego un movimiento de restitución (inciclotorsión, aducción y depresión), al desocluirlo. Suele ser más notorio en el ojo en abducción durante la lateroversión, cuando este es el ojo más comprometido, ya que es asimétrica. Además, la DVD puede ser clasificada como "compensada", cuando solo se desencadena por disociación de la imagen o desatención, y como "descompensada» cuando la desviación es constante o intermitente, lo cual ocurre en aproximadamente un $10 \%{ }^{1}$.

Presenta una asociación de casi el $70 \%$ a endotropías, y viene por lo general acompañada de nistagmus latente en el $50 \%$, según Anderson ${ }^{2}$. Puede también asociarse a hiperfunción de oblicuos inferiores en un $27.8 \%$, según Campomanes, et al. ${ }^{3}$, así como también a hiperfunción de los superiores en un 20 a 30\%, según Acosta-Silva ${ }^{4}$, y un $18.5 \%$, según Campomanes, et al. ${ }^{3}$, viéndose, entonces, implicado en los orígenes de algunos síndromes en $\mathrm{A}$ y en $\mathrm{V}^{1}$. Algo que se tiene claro acerca de la DVD es el alto riesgo de ambliopización en los casos descompensados. Es un fenómeno de participación cortical, cuyas causas no han sido del todo esclarecidas, aunque se han postulado múltiples teorías al respecto, y son de particular interés las teorías de Scobee (paresia bilateral de los rectos inferiores) ${ }^{5}$, de Posner (anomalía en el tono muscular debido a dominancia ocular patológica $)^{3}$ y de Bielschowsky (alteración de las vergencias verticales) ${ }^{6}$.

A pesar de que la DVD puede encontrarse en pacientes con binocularidad normal ${ }^{7}$, a menudo se encuentra asociada a endotropía congénita y, con menos frecuencia, a endotropía acomodativa, exotropía, y heterotropías de origen sensorial ${ }^{2}$. También se ha descrito asociada al síndrome de Duane ${ }^{8}$ y a la exotropía congénita, una anomalía poco frecuente ${ }^{9}$. Según Von
Noorden, la edad de diagnóstico de la DVD es entre los 2 y los 5 años, que es la edad en que se corrigen quirúrgicamente la mayoría de las desviaciones horizontales ${ }^{10}$.

La cirugía para la DVD ha variado mucho con el paso del tiempo, se han conservado algunos métodos, pero se han introducido otros aspectos innovadores para su manejo. Actualmente, el manejo depende en gran parte de la presencia o no de hiperfunción de los músculos oblicuos, al igual que de la agudeza y la capacidad visual, y también de la presencia o no de ambliopía. Con respecto al resultado quirúrgico, Romero-Apis reporta una disminución (sin desaparición) del grado de la DVD del $60 \%$ en uno de sus trabajos, con compensación total de la patología del $27 \%$, y regresión al grado original de la DVD del $10 \%{ }^{11}$. Con respecto a la retroinserción amplia de rectos superiores (RTRS), un estudio demostró que 23 de los 32 pacientes seleccionados para dicho estudio presentaron mejoría significativa estética de la desviación, después de un seguimiento de 3 años. En ninguno de estos pacientes se presentaron casos de paresia de los rectos superiores. La efectividad de la retroinserción de los rectos superiores en otros tipos de estrabismos verticales, en términos de corrección de la desviación en dioptrías prismáticas (DP) por milímetro de retroinserción, y el menor efecto de esta técnica en una DVD de la misma magnitud son algunos de los detalles que diferencian a esta patología de los demás tipos de estrabismos ${ }^{12}$.

En un reciente artículo, Pineles, et al. publicaron un estudio de 14 pacientes, a los cuales se les realizó transposición anterior del oblicuo inferior bilateral y de forma asimétrica, en los cuales obtuvieron una reducción marcada de la magnitud de la DVD, de un promedio preoperatorio de 18 DP hasta un promedio de 1 DP en el post-operatorio ${ }^{13}$. Existe un estudio mexicano del año 2007 de Arroyo-Yllanes, et al., con respecto al plegamiento de los rectos inferiores en el ojo con mala visión de 19 pacientes, de los cuales 10 presentaron disminución de la espontaneidad de la patología hasta 
1+, y los otros 9 presentaron disminución de la magnitud hasta $2+$, después del procedimiento quirúrgico, teniendo en cuenta que la mayoría tenía magnitudes de DVD de $4 t^{14}$.

\section{Objetivo}

Determinar el resultado posterior a la cirugía de la DVD descompensada en el Instituto de Oftalmología Fundación de Asistencia Privada Conde de Valenciana entre los años 2004 y 2014.

\section{Materiales y métodos}

Se realizó un estudio ambispectivo. Se tomaron los expedientes de todos los pacientes intervenidos quirúrgicamente para la corrección de la DVD entre los años 2004 y 2014 en el Servicio de Estrabismo del Instituto de Oftalmología Fundación Conde de Valenciana de la Ciudad de México, así como sus descripciones quirúrgicas. A todos los pacientes seleccionados que cumplían los criterios de inclusión se les citó para reevaluarlos y determinar sus variables actuales, con toma de fotos y video como constancia de su nueva evaluación.

Los procedimientos quirúrgicos para la corrección de la DVD realizados en el período de tiempo mencionado fueron: retroinserción de los rectos superiores (se realiza en ambos ojos, en pacientes con agudeza visual media binocular normal o por debajo de lo normal, con la desinserción de los músculos por encima del tendón muscular, y se retroinsertan simétrica o asimétricamente, dependiendo de la asimetría en la magnitud y espontaneidad de la DVD preoperatoria); plegamiento del recto inferior (para pacientes con espontaneidad de DVD en un ojo muy ambliope; se realiza una sutura cuyo efecto mecánico es plegar el músculo sobre sí mismo, entrando por un lado del tendón y saliendo por el otro, pasando por el cuerpo muscular a nivel de la marca dejada por una maniobra de plegamiento que se realiza con los ganchos de estrabismo, denominada «maniobra del beso y del abrazo», y finalmente anudando a nivel del tendón); y transposición anterior del oblicuo inferior (para pacientes con DVD asociada a hiperfunción del oblicuo inferior; se desinserta el músculo a nivel de su tendón y se transpone con sutura junto al recto inferior).

Debido a que en la retroinserción de los rectos superiores, la gran mayoría de las intervenciones fue simétrica (23 de 29), se decidió agrupar en un solo grupo las retroinserciones del recto superior simétricas y asimétricas. Del mismo modo, debido a que la muestra para los otros dos tipos de intervenciones quirúrgicas fue muy pequeña, también se decidieron agrupar los plegamientos del recto inferior monoculares y binoculares en un grupo, y se procedió de igual forma con las transposiciones anteriores de los oblicuos inferiores.

Nuestras variables de interés fueron: edad en el momento de la cirugía, sexo, presencia de ambliopía, patología estrabológica asociada, magnitud preoperatoria de la desviación, cirugía realizada, resultado quirúrgico (compensado si no hubo manifestación espontánea de la DVD en la visión binocular, o descompensado si la DVD se manifestó de forma espontánea en la visión binocular), requerimiento de más cirugías y tiempo de seguimiento.

Los criterios de inclusión fueron: 1) pacientes que entre los años 2004 a 2014 fueron intervenidos para tratamiento quirúrgico de la DVD en la institución, y 2) pacientes que hubiesen sido reintervenidos por DVD residual en la institución. Los criterios de exclusión fueron: 1) pacientes que fueron intervenidos para el manejo quirúrgico de la DVD que presenten patología de tipo parética asociada contralateral, por riesgo de incurrir en un sesgo de selección, p.ej., parálisis/paresia del oblicuo inferior asociada a DVD; 2) pacientes con expediente incompleto, y 3) pacientes que no hubiesen cumplido un seguimiento de por lo menos 6 meses.

La magnitud de la DVD se midió usando pantalleo monocular en la visión lejana y neutralizando cualquier desviación horizontal (en caso de presencia de alguna), posteriormente usando el sistema de "cruces» (+ = aproximadamente menor que $10 \mathrm{DP},++=$ aproximadamente menor que $20 \mathrm{DP},+++=$ aproximadamente entre 20 y $30 \mathrm{DP}, \mathrm{y}++++=$ aproximadamente mayor que $30 \mathrm{DP}$ ). Además y muy importante, se tuvo en cuenta si en la visión binocular el paciente presentaba espontaneidad de la DVD. La «no espontaneidad» de la DVD en el momento de la revisión de los pacientes del estudio se tomó como resultado quirúrgico «compensado", y la espontaneidad como resultado quirúrgico «descompensado».

La muestra se obtuvo teniendo en cuenta el porcentaje de recidivas de la técnica quirúrgica utilizada. Se tomó un alfa (error tipo I) de 0.05 y un error b (error tipo II) del 10\% (poder del 90\%). La muestra estuvo formada por 50 pacientes para el grupo estudio.

Se realizó un análisis descriptivo de todas las variables de interés (frecuencias y porcentajes), así como la estimación de desviaciones estándar, medianas y modas. Se utilizó el sistema SPSSv15 para 

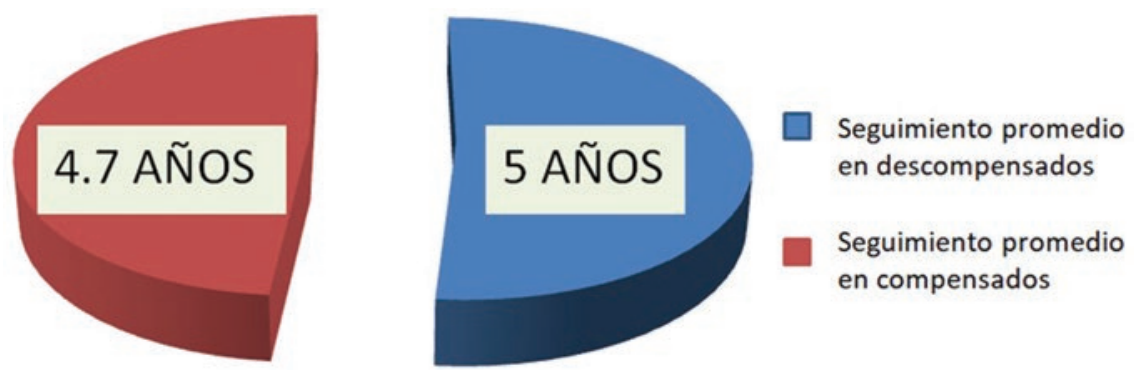

Figura 1. Relación entre tiempo de seguimiento y resultado quirúrgico.

determinar las asociaciones estadísticamente significativas, teniendo en cuenta que $p<0.05$. Dicho análisis incluyó la prueba exacta de Fisher para la comparación de proporciones de las variables categóricas, y la prueba de Kruskal-Wallis y la correlación de Pearson para las variables continuas.

\section{Resultados}

Se estudiaron 40 pacientes. La edad promedio en el momento de la cirugía fue de 9.625 años, con una desviación estándar de más o menos 7 años. La moda fue de 4 años. La mediana fue de 7.5 años. La mayoría de los pacientes de este trabajo pertenecían al sexo femenino (70\%), y tan solo 12 pacientes, al sexo masculino (30\%).

El $65 \%$ de los pacientes de este estudio presentaban endotropía no acomodativa como patología asociada, y el $32.5 \%$ de los pacientes presentaban desviación horizontal disociada como patología asociada. La suma de las demás patologías apenas llega al 2.5\% ETPA (endotropía parcialmente acomodativa).

Al $77.5 \%(n=31)$ de los pacientes de este estudio se le realizó retroinserción de ambos rectos superiores, comparado con los otros métodos (transposición anterior de ambos oblicuos inferiores menos del 10\%, plegamiento de recto(s) inferior(es) del $15 \%$ ).

El promedio de tiempo de seguimiento fue 4.75 años, con una desviación estándar más o menos de 2.244 años, y la mediana para esta variable fue de 5 años (Fig. 1). Del total de los pacientes del estudio, un $87.5 \%$ $(n=35)$ presentó compensación de la DVD durante el tiempo de seguimiento postoperatorio. En solo 5 pacientes no se compensó la DVD después de la cirugía (12.5\%).

En total, se hallaron 12 pacientes ambliopes y 28 pacientes no ambliopes (70\% este último). De los pacientes ambliopes, 9 se hallaron con desviaciones

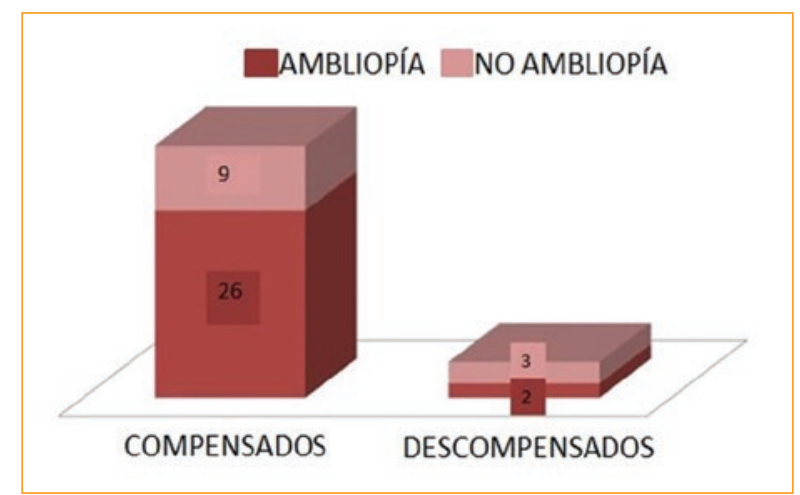

Figura 2. Relación entre resultado quirúrgico y capacidad visual postoperatoria (en términos de presencia de ambliopía).

compensadas al final del estudio, y 3 , descompensadas; de los pacientes ambliopes, se encontraron 10 con ambliopía unilateral (2 del ojo derecho y los demás, del izquierdo) y 2 con ambliopía bilateral. De los pacientes no ambliopes, 26 se hallaban compensados y 2, descompensados. Esta relación no fue estadísticamente significativa ( $p=0.118$ ) (Fig. 2).

La edad promedio de los pacientes que no mejoraron la magnitud de la DVD y se mantuvieron descompensados fue de 5.4 años, con una desviación estándar de más o menos 1.02 años, con una mediana de 5 años. El promedio de edad de los pacientes que sí mejoraron fue de 10.22 años, con una desviación estándar de más o menos 7.27 años, con una mediana de 8 años. Esta relación no fue estadísticamente significativa ( $p=0.1568)$.

El procedimiento para el tratamiento de la DVD que más se realizó en los años comprendidos entre 2004 y 2014 fue la retroinserción de los rectos superiores, con un porcentaje de descompensación del 13\% de la totalidad de los que se les realizó este procedimiento. E 


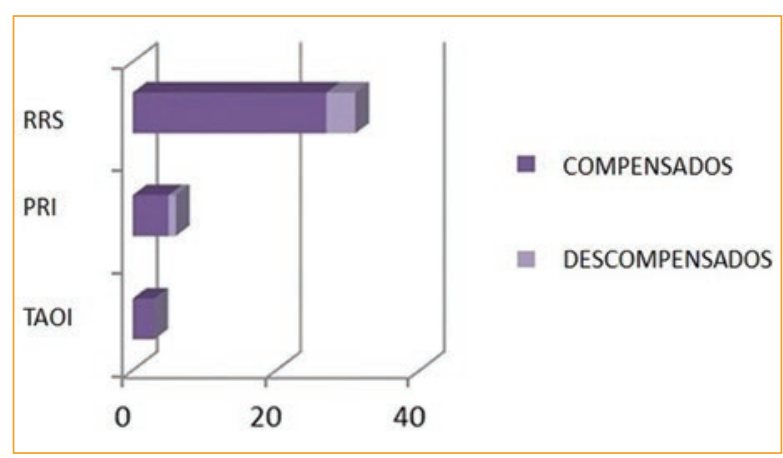

Figura 3. Relación entre tipo de cirugía y resultado quirúrgico. PRI: plegamiento de los rectos inferiores;

RTRS: retroinserción amplia de los rectos superiores;

TAOI: transposición anterior del oblicuo inferior bilateral.

plegamiento de rectos inferiores se realizó en 6 pacientes con un porcentaje de descompensación del 15\%. A los que se les realizó la transposición anterior de los oblicuos inferiores, no presentaron porcentajes de descompensación (Fig. 3). Además, se encontró que aquellos a quienes se les realizó la retroinserción de rectos superiores y quedaron descompensados, requirieron en promedio 1.5 cirugías adicionales, y que no presentan aún compensación, a pesar de estos. Hubo un caso de hipercorrección después del plegamiento del recto inferior. Esto no fue estadísticamente significativo ( $p=0.768)$.

De los pacientes sin ambliopía, 19 eran del sexo femenino y 8 , del masculino. De los pacientes ambliopes, 8 eran del sexo femenino y 4 , del masculino. En relación con el resultado postoperatorio, de los 34 pacientes compensados, 11 eran del sexo masculino, y 23 , del femenino. Esta relación no fue estadísticamente significativa $(p=0.602)$. De los pacientes descompensados, 1 era del sexo masculino, y 4 , del femenino (Fig. 4).

\section{Discusión}

De acuerdo con la literatura consultada para este trabajo, como el trabajo de Romero-Apis del año 1971, la compensación de la DVD después de su cirugía correctora puede alcanzar el $60 \%$ aproximadamente ${ }^{11}$. En este trabajo se obtuvo una compensación del $80 \%$. Es probable que en esto tenga que ver, tal vez, el refinamiento de las técnicas quirúrgicas con el paso del tiempo y el tamaño de la muestra. Nuestro resultado también estuvo muy acorde con lo hallado por Esswein, et al. ${ }^{12}$. No obtuvimos diferencias estadísticamente significativas con respecto al resultado y al tipo de cirugía.

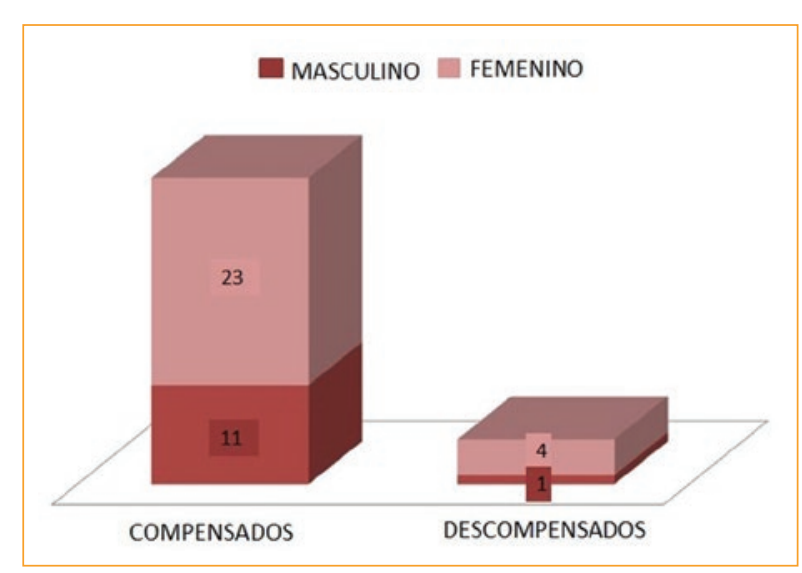

Figura 4. Relación entre el sexo y el resultado quirúrgico.

A la mayoría se les realizó retroinserción retroecuatorial simétrica de ambos rectos superiores, debido a que presentaban criterios para llevar esta cirugía a cabo, es decir, no presentaban ambliopía profunda en uno de los ojos, o presentaban ambliopía simétrica en los pocos casos que hubo en este trabajo. Los pacientes de este estudio fueron bien seleccionados para el tipo de cirugía que se debía hacer. Hubo 2 pacientes ambliopes unilaterales a quienes se les realizó retroinserción de un recto superior, una con ambliopía moderada. De acuerdo al trabajo de Arroyo-Yllanes, et al., en los casos en los cuales se tiene una ambliopía muy profunda unilateral, el mejor tratamiento (y a su vez, el menos invasivo) es el plegamiento del recto inferior del ojo ambliope con DVD descompensada ${ }^{14}$.

En el presente trabajo tuvimos 4 pacientes que requirieron una segunda cirugía por persistencia de la DVD descompensada después de la retroinserción de rectos superiores, lo cual es muy similar a lo encontrado por Magoon, et al., aunque en este estudio se estaban evaluando únicamente los resultados de la corrección de la DVD descompensada por medio de cirugía asimétrica en los rectos superiores (basándose, según estos autores, en el hecho de que la DVD se manifiesta de forma asimétrica, y sin tener en cuenta la agudeza visual de los pacientes para la toma de decisiones) ${ }^{15}$.

Tuvimos una tasa de hipercorrecciones baja, comparada con la que se muestra en el estudio de Braverman, et al. Ellos presentaron 4 casos de hipercorrección asociada a la cirugía de los rectos superiores, mientras que las que se obtuvieron en el presente estudio fueron posteriores a la cirugía del recto inferior ${ }^{16}$.

Algo para tener en cuenta es que, en este trabajo, no hubo riesgo de sesgo de selección para la variable de seguimiento, ya que los pacientes de diferentes 
años de seguimiento se hallaban repartidos ecuánimemente entre los grupos de compensados y descompensados, y no encontramos publicaciones que tuviesen en cuenta esta distinción.

Este estudio pudo haber sido un estudio retrospectivo simple, pero se decidió hacerlo lo más actualizado posible, al citar pacientes que en la mayoría de los casos se habían perdido en su seguimiento durante más de 2 años, para volver a valorar su estado actual, con el respaldo de las fotos y los videos, lo que lo hace un estudio con raíces retrospectivas, pero con un análisis descriptivo y al día. La falencia de este estudio recae sobre su muestra un poco pequeña, especialmente aquella de pacientes manejados con plegamiento del recto inferior y transposición anterior del oblicuo inferior, aunque confiamos en que esto no habría cambiado nuestros resultados.

\section{Conclusiones}

El éxito de la cirugía para la corrección de la DVD es alta en nuestra institución, con valores más altos que en estudios previos publicados, y la cirugía que más se realiza en la institución es la retroinserción de rectos superiores. El plegamiento de rectos inferiores se realiza con menor frecuencia, de acuerdo al tamaño de la nuestra muestra. La presencia de ambliopía no determinó en este estudio el éxito o el fracaso de las cirugías, al no ser estadísticamente significativa esta asociación. La edad de realización de la corrección quirúrgica tampoco se asoció a resultados estadísticamente significativos. Si la muestra fuese mayor, probablemente habría cambios con respecto a la frecuencia, pero no con respecto al éxito quirúrgico. No hubo relación entre las patologías asociadas a la DVD con el éxito quirúrgico a mediano y largo plazo. Es el primer estudio del que se tenga conocimiento en este país en el cual se comparen técnicas quirúrgicas, capacidad visual postoperatoria, diagnósticos asociados, resultados y seguimiento postoperatorio para determinar los perfiles de éxito de la corrección quirúrgica de la DVD, y es necesario que se realicen más estudios de esta índole en otras instituciones, para determinar cuál es la tendencia poblacional en lo que a resultados después de la cirugía de DVD respecta.

\section{Conflicto de intereses}

Los autores declaran no tener conflictos de intereses.

\section{Responsabilidades éticas}

Protección de personas y animales. Los autores declaran que para esta investigación no se han realizado experimentos en seres humanos ni en animales.

Confidencialidad de los datos. Los autores declaran que en este artículo no aparecen datos de pacientes.

Derecho a la privacidad y consentimiento informado. Los autores declaran que en este artículo no aparecen datos de pacientes.

\section{Bibliografía}

1. Romero-Apis D, Castellanos-Bracamontes A, Acosta-Silva M. Estrabismos disociados. En: Temas Selectos de Estrabismo. p. 49-60.

2. Kutluk S, Avilla CW, von Noorden GK. The prevalence of dissociated vertical deviation in patients with sensory heterotropia. Am J Ophthalmol. 1995; $119: 744$

3. Campomanes-Eguiarte G, Castellanos-Bracamontes A, González y Gutiérrez L. Desviación vertical disociada. En: Actualidades del Estrabismo Latinoamericano. Congreso Latinoamericano de Estrabismo, CIBA Vision. 1998;241-52.

4. Acosta-Silva M. Cirugía de cuatro oblicuos para DVD y síndrome en "A". En: Temas Selectos de Estrabismo. 2. ${ }^{a} e d .2005 ;$ p. 125.

5. Scobee RG. The oculorotary muscles. St. Louis: The CV. Mosby and Co., 1947; p. 145.

6. Bielschowsky A. Disturbances of the vertical motor muscles of the eye. Arch Ophthalmol. 1938;20:175-200.

7. Van Rijn LJ, ten Tusscher MP, de Jong I, Hendrikse F. Assymetrical vertical phorias indicating dissociated vertical deviation in subjects with normal binocular vision. Vision Res. 1998;38(19):2973-8.

8. Rimmer S, Katz B. Dissociated vertical deviation in a patient with Duane's retraction syndrome. J Clin Neuro Ophthalmol. 1990;10:38.

9. Metz HS, Norris H. Cyclotorsional diplopia following retinal detachment surgery. J Pediatr Ophthalmol Strabismus. 1987;24:287.

10. Von Noorden GK, Campos EC. Cyclovertical Deviations. En: Binocular vision and ocular motility. Theory and management of strabismus. $6^{\text {th }}$ ed. Mosby, 2002; p. 378-85.

11. Romero-Apis D. Early surgery results in strabismus. J Pediatr Ophthalmol. 1971:8:93.

12. Esswein MB, von Noorden GK, Coburn A. Comparison of surgical methods in the treatment of dissociated vertical deviation. Am J Ophthalmol. 1992;113:287.

13. Pineles SL, Vélez G, Vélez FG. Asymmetric inferior oblique anterior transposition for incommitant asymmetric dissociated vertical deviation. Graefes' Arch Ophthalmol. 2013;251(11):2639-42.

14. Arroyo-Yllanes ME, Escanio-Cortés ME, Pérez-Pérez JE, Murillo-MuriIlo L. Plegamiento del recto inferior unilateral para el tratamiento de la desviación vertical disociada. Cirugía y cirujanos. 2007;75(1):7-12.

15. Magoon E, Cruciger M, Jampolsky A. Dissociated vertical deviation: an asymmetric condition treated with large bilateral superior rectus recession. J Pediatr Ophthalmol Strabismus. 1982;19(3):152-6.

16. Braverman DE, Scott WE. Surgical correction of dissociated vertical deviations. J Pediatr Ophthalmol. 1977;14(6):337-42. 